\title{
Electroplating of Copper-Based Alloys for the Catalytic Conversion of $\mathrm{CO}_{2}$
}

\author{
Lee Fuller ${ }^{1}$, Jason Martin, ${ }^{1}$ Seth King, ${ }^{2}$ and Sujat Sen ${ }^{3}$ \\ ${ }^{I}$ Student (Department of Chemistry and Biochemistry, University of Wisconsin La Crosse, La- \\ Crosse WI, USA) \\ ${ }^{2}$ Professor (Department of Physics, University of Wisconsin La Crosse, La Crosse, WI, USA) \\ ${ }^{3}$ Professor (Department of Chemistry and Biochemistry, University of Wisconsin La Crosse, La- \\ Crosse, WI, USA)
}

\begin{abstract}
A variety of materials have been explored in the literature, but metals such as copper have been shown to uniquely generate appreciable amounts of hydrocarbon products during the electrocatalytic conversion of carbon dioxide $\left(\mathrm{CO}_{2}\right)$. Furthermore, alloying copper with other metals such as tin has also been demonstrated to tune the selectivity towards more desirable products and minimize the parasitic hydrogen evolution reaction. Herein, we describe the use of pulsed current methods to successfully electrodeposit particles of copper-tin alloys on the surface of commercially available gas diffusion layer (GDL) substrates. Images obtained from an optical microscope were used to qualitatively assess the uniformity of the deposited particles. A scanning electron microscope (SEM) was used to qualitatively assess the size of these particles, which varied from micron-sized chunks to finer sub-micron particles. Additionally, x-ray diffraction (XRD) was used to identify the phase and composition of the alloys.
\end{abstract}

\section{Introduction}

Copper $(\mathrm{Cu})$ is a well-known catalyst for the electrochemical $\mathrm{CO}_{2}$ reduction reaction $\left(\mathrm{eCO}_{2} \mathrm{RR}\right)$ known to produce a wide variety of products, including formic acid, methane, ethylene, and carbon monoxide (Peterson et al., 2010). Some of these chemicals can be used as precursors for plastics and petrochemicals, while others are used as fuels that store energy. When these fuels are burnt for energy, more $\mathrm{CO}_{2}$ is produced, which can be fed back into the $\mathrm{eCO}_{2} \mathrm{RR}$, creating a sustainable $\mathrm{CO}_{2}$ recycling system. Electrochemical $\mathrm{CO}_{2}$ reduction is advantageous compared to other methods of converting $\mathrm{CO}_{2}$ because it can be conducted at ambient conditions, and is easy to control the selectivity. Most importantly, electrons are cheap, clean, and efficient (Yin, Palmore, \& Sun, 2019).

Beginning in the 1980s Hori et al. (Hori, Murata, \& Takahashi, 1989; Hori, Wakebe, Tsukamoto, \& Koga, 1994) have demonstrated that many different metals are excellent catalysts for the $\mathrm{eCO}_{2} \mathrm{RR}$ and classified them depending on the majority product formed. For example, metals such as $\mathrm{Au}, \mathrm{Ag}, \mathrm{Zn}, \mathrm{Pd}$, and $\mathrm{Ga}$ are selective towards carbon monoxide; Metals such as $\mathrm{Pb}, \mathrm{Hg}, \mathrm{In}, \mathrm{Sn}$, $\mathrm{Cd}$, and $\mathrm{Tl}$ are selective towards formate; and metals such as $\mathrm{Ni}, \mathrm{Fe}, \mathrm{Pt}$, and Ti are selective towards hydrogen, which is a competing reaction in aqueous media. Copper $(\mathrm{Cu})$ is unique because it produces a wide range of products, including methane, ethylene, ethanol, propanol, carbon monoxide, and formate (Peterson et al., 2010). Since $\mathrm{Cu}$ is not selective towards one specific product, it is not an ideal catalyst by itself for large scale applications where separation costs can be high. However, $\mathrm{Cu}$ can be alloyed with other metals such as Tin (Sn) to create a catalyst that is more selective towards one product (Katoh, Uchida, Shibata, \& Watanabe, 1994).

The size of the particle is also known to have a significant effect on catalytic performance (Sen et al., 2014). As the particle size decreases, the surface area per unit volume increases, and the effective catalytically active surface area increases. Since the $\mathrm{eCO}_{2} \mathrm{RR}$ is a heterogeneous process, a larger catalytic surface area per unit volume is beneficial. To create nanostructures, the literature has reported several traditional solution-phase methods, which are then physically deposited onto 
a substrate (Sen, et al., 2019). Herein, we have investigated the use of electrodeposition as a means to directly generate and deposit nanostructures of metals on the surface of a gas diffusion layer (GDL) substrate. One form of electrodeposition is pulse current (PC) electrodeposition (Chandrasekar \& Pushpavanam, 2008; Sen et al., 2016). Figure 1 shows a typical waveform as a function of time.

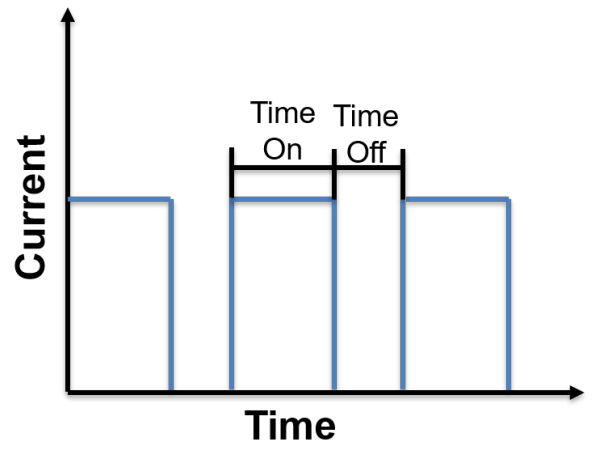

Figure 1. Current vs. time profile for pulsed current (PC) electrodeposition.

In PC electrodeposition, a constant current is applied for a certain amount of time (time-on), followed by a rest where no current is applied (time-off). After one cycle completes, another cycle begins, and this repeats until specific parameters such as current density and charge density are met. This is distinctly different from direct current (DC) electrodeposition that involves a constant current for longer durations that typically lead to large micron sized deposits (Katoh et al., 1994). Herein, we demonstrate the use of pulsed current to electrodeposit alloy particles with a diameter smaller than 1 micron, and in select cases, particles with a fine structure smaller than $100 \mathrm{~nm}$ with a relatively uniform coverage of the substrate.

\section{Materials and Methods}

Substrate. In this study, the catalyst was deposited on a commercially available Sigracet 39BC gas diffusion layer (GDL) substrate (Fuel Cell Store). This substrate has two main layers, namely the microporous layer (MPL) with an approximate thickness of 10 microns and the Carbon fiber substrate (CFS) underneath with an approximate thickness of 300 micron. The manufacturer specifies a Teflon concentration of $23 \%$ in the MPL.
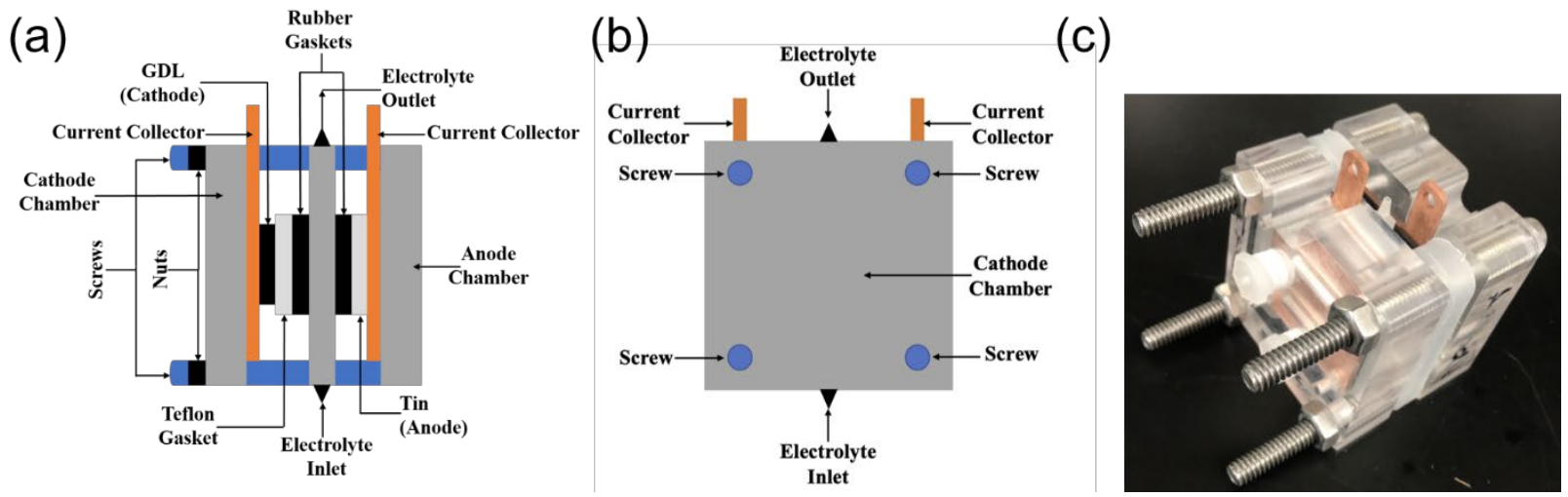
For each experiment, a $2.5 \mathrm{~cm}$ by $2.5 \mathrm{~cm}$ square was cut from the stock GDL sheet. The mass of each GDL was recorded before deposition and then again after deposition in order to determine the mass deposited during electrodeposition.

Flow Cell Electrodeposition Setup. The experimental set-up shown schematically in Figure 3 consists of a flow cell, a peristaltic pump (MasterFlex L/S Peristaltic Pump, EW- 7792165), a power supply (Dynatronix, MicroStar Series, Model Number: DuPR10-3-6 XR), argon gas (UHP Grade, Mississippi Welder Supply Co, Inc.) and an electrolyte reservoir. The flow cell is made up of a cathode chamber, anode chamber, electrolyte chamber, gaskets for sealing, a GDL, a tin counter electrode, and screws and nuts adapted from a similar design (Sen et al., 2019).

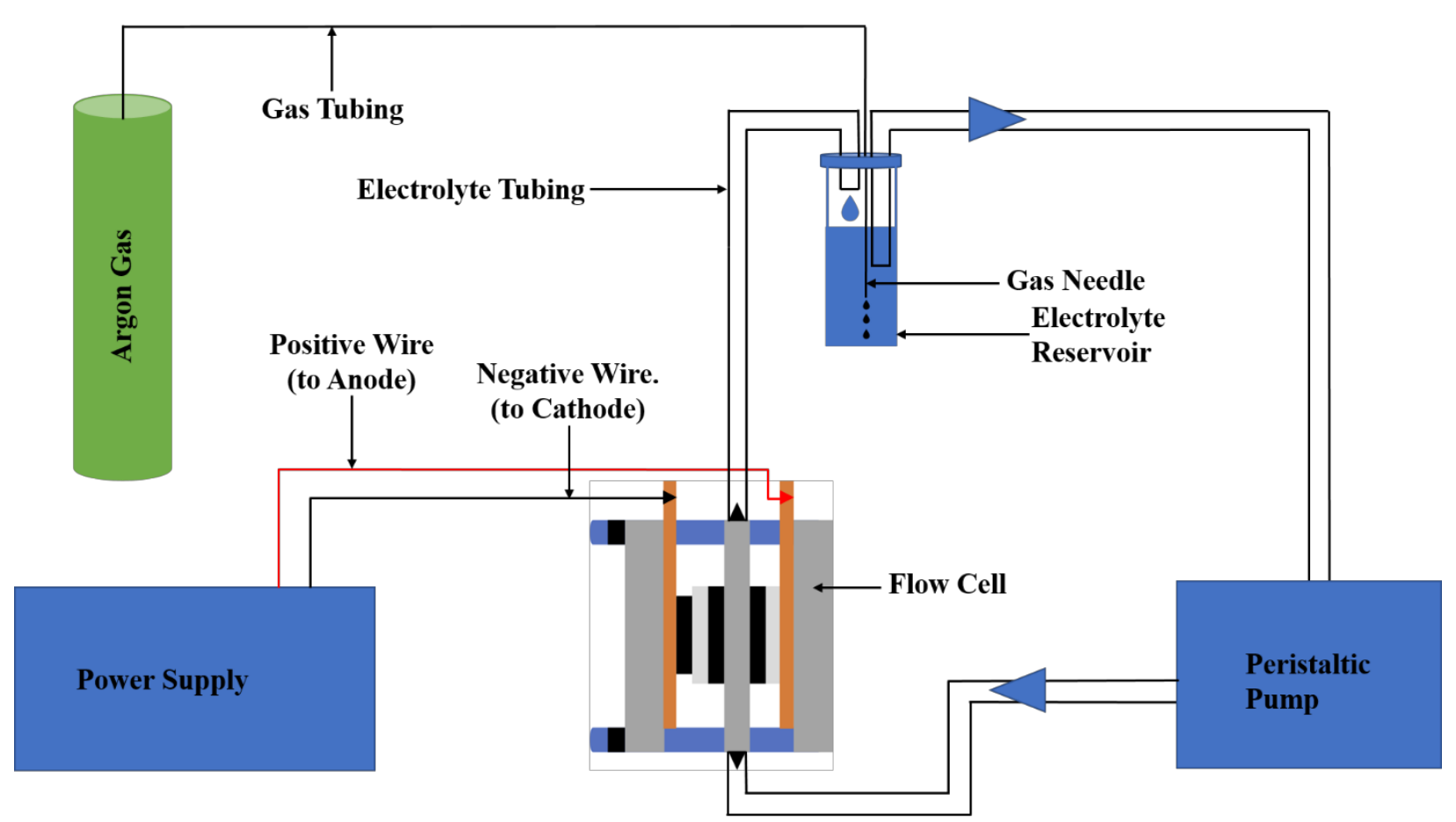

Figure 3. Schematic of the flow cell electrodeposition experimental setup.

Electrodeposition Solution. The electrodeposition solution was made by first diluting methanesulfonic acid (MSA) (Sigma-Aldrich 70 wt. \% in $\mathrm{H}_{2} \mathrm{O}$ ) three-fold. Copper (II) sulfate pentahydrate $\left(\mathrm{CuSO}_{4} \cdot 5 \mathrm{H}_{2} \mathrm{O}\right)$ (Sigma-Aldrich) and tin (II) methanesulfonate (Sn-MSA) (SigmaAldrich 50 wt. $\%$ in $\mathrm{H}_{2} \mathrm{O}$ ) were dissolved in the diluted MSA to create a $0.1 \mathrm{M} \mathrm{CuSO} \cdot 5 \mathrm{H}_{2} \mathrm{O}$ and $0.1 \mathrm{M}$ Sn-MSA solution with a $\mathrm{pH}$ of 0.72 . These procedures were adapted from the work of Low et al. (Low \& Walsh, 2008)

Run-Time Calculations. Primary variables involved in PC electrodeposition include charge density at the cathode $\left(\mathrm{C} / \mathrm{cm}^{2}\right)$, the current density at the cathode $\left(\mathrm{A} / \mathrm{cm}^{2}\right)$, time that current is applied in one cycle (time-on), and time that current is not applied during the same cycle (timeoff) as shown in figure 1 . To satisfy the four parameters, a calculation is made to solve for the total-run time, which is shown below. Additionally, a sample calculation is shown. 
Electrodeposition Parameters:

Charge Density: $x \mathrm{C} / \mathrm{cm}^{2}$

Current Density: $y \mathrm{~A} / \mathrm{cm}^{2}$

Active Surface Area: $4 \mathrm{~cm}^{2}$

Time-On: $a$ seconds

Time-Off: $b$ seconds

1 Cycle $=$ Time-On + Time-Off $=(a+b)$ seconds

1. Charge Density $\bullet$ Active Surface Area $=$ Total Charge $x \mathrm{C} / \mathrm{cm}^{2} \cdot 4 \mathrm{~cm}^{2}=4 x \mathrm{C}$

2. Current Density $\bullet$ Active Surface Area $=$ Total Current $y \mathrm{~A} / \mathrm{cm}^{2} \cdot 4 \mathrm{~cm}^{2}=4 y \mathrm{~A}$

3. $1 \mathrm{C}$ is equal to $1 \mathrm{~A} \cdot 1 \mathrm{~s}$. Therefore, given total current and charge, time $(t)$ can be solved for:

$4 x \mathrm{C}=4 y \mathrm{~A} \cdot t$

$t=\frac{4 x \mathrm{C}}{4 y \mathrm{~A}}$

$t=\frac{4 x \mathrm{~A} \cdot \mathrm{s}}{4 y \mathrm{~A}}$

$t=\frac{x}{y} \mathrm{~s}$

4. From there, $t$ is divided by the time-on value to solve for the total amount of cycles required.

$\frac{x}{y} \mathrm{~s} \div a \mathrm{~s}=\frac{x}{y a}$ cycles

5. The amount of time for one cycle to complete (time-on + time-off) is multiplied by the number of total cycles to find the total run-time for electrodeposition.

$\frac{x}{y a} \cdot(a+b) \mathrm{s}=\frac{x(a+b)}{y a} \mathrm{~s}$

Therefore, the total run-time for PC electrodeposition is $\frac{x(a+b)}{y a}$ seconds. A Sample Calculation is shown below:

Charge Density: $1 \mathrm{C} / \mathrm{cm}^{2}$

Current Density: $0.1 \mathrm{~A} / \mathrm{cm}^{2}$

Active Surface Area: $4 \mathrm{~cm}^{2}$

Time-On: 0.01 seconds

Time-Off: 0.09 seconds

$1 \mathrm{C} / \mathrm{cm}^{2} \cdot 4 \mathrm{~cm}^{2}=4 \mathrm{C}$

$0.1 \mathrm{~A} / \mathrm{cm}^{2} \cdot 4 \mathrm{~cm}^{2}=0.4 \mathrm{~A}$

$4 \mathrm{C}=0.4 \mathrm{~A} \cdot t$

$t=\frac{4 \mathrm{C}}{0.4 \mathrm{~A}}=\frac{4 \mathrm{~A} \cdot \mathrm{s}}{0.4 \mathrm{~A}}=10 \mathrm{~s}$

$10 \mathrm{~s} \div 0.01 \mathrm{~s}=1000$ cycles

$1000 \cdot 0.1 \mathrm{~s}=100 \mathrm{~s}$

Therefore, the total run-time for this experiment is 100 seconds.

Pre-Electrodeposition Protocol. A $2.5 \mathrm{~cm}$ x $2.5 \mathrm{~cm}$ piece of Tin (Sn, 99.9\%, McMaster Carr) and the two copper current collectors were sanded with fine sandpaper and put in a sonicator 
for 10 minutes. The Tin served as the anode, while a GDL served as the cathode of the flow cell. After the current collectors and the Tin anode were cleaned, the flow cell was assembled using 14 lbf.in torque applied with an EMT50 Torque screwdriver (Mountz Inc.), as shown in figure 3. After assembly, it was clamped to a ring stand and electrolyte was pumped through it for 10 minutes at $1 \mathrm{~mL} / \mathrm{min}$ using a pump. Simultaneously, argon gas was purged the electrolyte reservoir for 10 minutes, before it was connected to the DuPR. The negative terminal of the DuPR attaches to the cathode current collector and the positive terminal of the DuPR attaches to the anode current collector. Finally, the electrodeposition parameters were entered into the DuPR.

Electrodeposition Parameters. PC electrodeposition was used to deposit copper-tin alloys onto GDL's, as a function of varying current density (see Table 1).

Table 1. Parameters for PC electrodeposition of copper-tin alloys.

\begin{tabular}{|c|c|c|c|c|}
\hline $\begin{array}{c}\text { Current Density } \\
\left(\mathbf{m A} / \mathbf{c m}^{\mathbf{2}}\right)\end{array}$ & $\begin{array}{c}\text { Charge Density } \\
\left(\mathbf{C} / \mathbf{c m}^{\mathbf{2}}\right)\end{array}$ & Time-On (ms) & Time-Off (ms) & Duty Cycle (\%) \\
\hline 10 & 6.25 & 10 & 90 & 10 \\
\hline 100 & 6.25 & 10 & 90 & 10 \\
\hline 250 & 6.25 & 10 & 90 & 10 \\
\hline 500 & 6.25 & 10 & 90 & 10 \\
\hline
\end{tabular}

Mass Loading and Thickness. After electrodeposition was completed, the GDL was soaked in DI water for 5 minutes to remove excess electrodeposition solution such as residual copper or tin ions, and subsequently let out to dry in air overnight. After drying, the electrodeposited GDL was weighed in order to determine how much mass was deposited during the experiment. The mass loading of each trial was found by subtracting the mass of the GDL before electrodeposition from the mass of the GDL after electrodeposition. This value was then divided by 2.55 (area of deposition is $2.55 \mathrm{~cm}^{2}$ ) to obtain mass loading $\left(\mathrm{mg} / \mathrm{cm}^{2}\right)$. Additionally, pictures of the electrodeposited GDL's were taken (Digital Microscope) in order to compare the color and coverage of various electrodeposition trials. Finally, the approximate thickness of these films was also determined in the range of 2 - 5 microns using a Tencorp P-7 Stylus profiler. Additionally, porosity and thickness of these films can play an important role in their ability to act as good catalysts, which is a subject of future investigation.

Imaging. A scanning electron microscope (SEM) (Zeiss, EVO HD 15) was used to determine the size of electrodeposited particles on GDL's. The typical working distance used to image was $9.5 \mathrm{~mm}$, and the typical accelerating voltage was $10.0 \mathrm{kV}$. An Amscope (Model \# H800-96S-AF22) Digital inspection microscope was used to obtain the optical images.

X-ray Diffraction. An X-ray diffractometer (XRD) (Siemens D500, Cu K-alpha, BraggBrentano geometry) was used to determine the elemental composition of catalysts created by scanning at 0.05 degrees per step, dwell time of $4 \mathrm{~s}$ from 20 to 90 degrees. Match3 and ICSD databases were used to compare the experimental patterns obtained and assign phase identification.

\section{Experimental Results}

Microscopy. Figure 4 shows the optical images of the substrates as a function of varying current density after drying overnight. At the lowest current density (4 (a), $10 \mathrm{~mA} / \mathrm{cm}^{2}$ ), the visible golden-brown color clearly suggests the deposition of metallic copper as opposed to an alloy. As 
current density was increased from 10 to $500 \mathrm{~mA} / \mathrm{cm}^{2}$, a clear transition from golden brown to grey-black color was observed, suggesting a gradual change in the phase of the electrodeposited material.
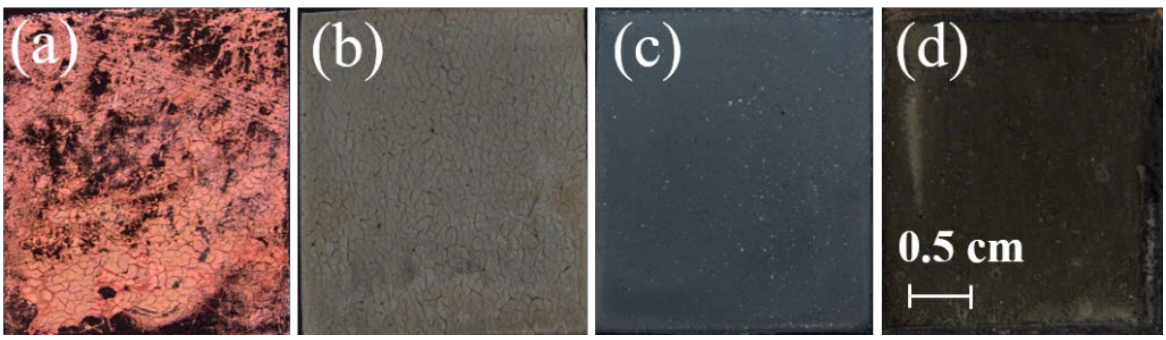

Figure 4. Optical images of catalysts deposited at varying current densities (scale bar included). The current densities for each image are as follows: a) $10 \mathrm{~mA} / \mathrm{cm}^{2}$, b) $100 \mathrm{~mA} / \mathrm{cm}^{2}$, c) $250 \mathrm{~mA} / \mathrm{cm}^{2}$, d) $500 \mathrm{~mA} / \mathrm{cm}^{2}$.

SEM images of the same electrodeposited samples were also obtained as shown in figure 5 at low and high magnifications. Deposits created at current densities of 250 and $500 \mathrm{~mA} / \mathrm{cm}^{2}$ look especially promising since discrete particles were observed with individual particle size much smaller than 200 nanometers in diameter, which is ideal for heterogeneous catalytic applications such as the $\mathrm{eCO}_{2} \mathrm{RR}$.
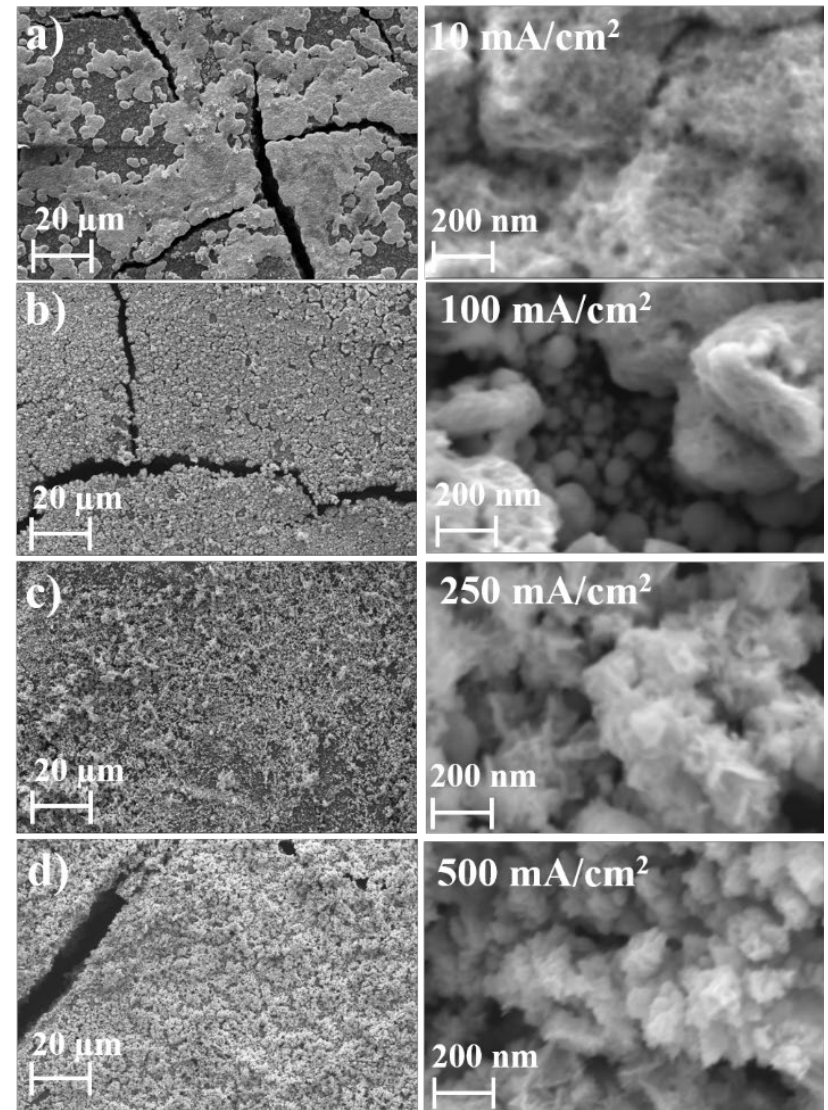

Figure 5. SEM images (scale bars included) of catalysts corresponding to figure 4 . The current densities for each image are as follows: a) $10 \mathrm{~mA} / \mathrm{cm}^{2}$, b) $100 \mathrm{~mA} / \mathrm{cm}^{2}$, c) $250 \mathrm{~mA} / \mathrm{cm}^{2}$, d) $500 \mathrm{~mA} / \mathrm{cm}^{2}$. 
X-ray Diffraction. As shown in figure 6, XRD was performed on all samples in order to confirm the phase and composition of the electrodeposited material. The pristine (uncoated) GDL was found to have a large peak at approximately 26 degrees, likely arising from the graphite present in the microporous layer (MPL). This was used as a reference peak to verify remaining patterns also shown in figure 6 . The XRD patterns for the deposit at a current density of $10 \mathrm{~mA} / \mathrm{cm}^{2}$ matched the reference database pattern of pure copper perfectly, as indicated by the *, thereby confirming the initial observation of a copper-color deposit. The catalysts created at current densities of $250 \mathrm{~mA} / \mathrm{cm}^{2}$ and greater all matched well with database standards for the known $\mathrm{Cu}_{6} \mathrm{Sn}_{5}$ alloy. The XRD pattern of the catalyst created at $100 \mathrm{~mA} / \mathrm{cm}^{2}$ did not match the patterns for pure copper metal or the $\mathrm{Cu}_{6} \mathrm{Sn}_{5}$ alloy, and suggests the formation of a different atypical alloy composition. A database match was not found, and will be the subject of future investigation.

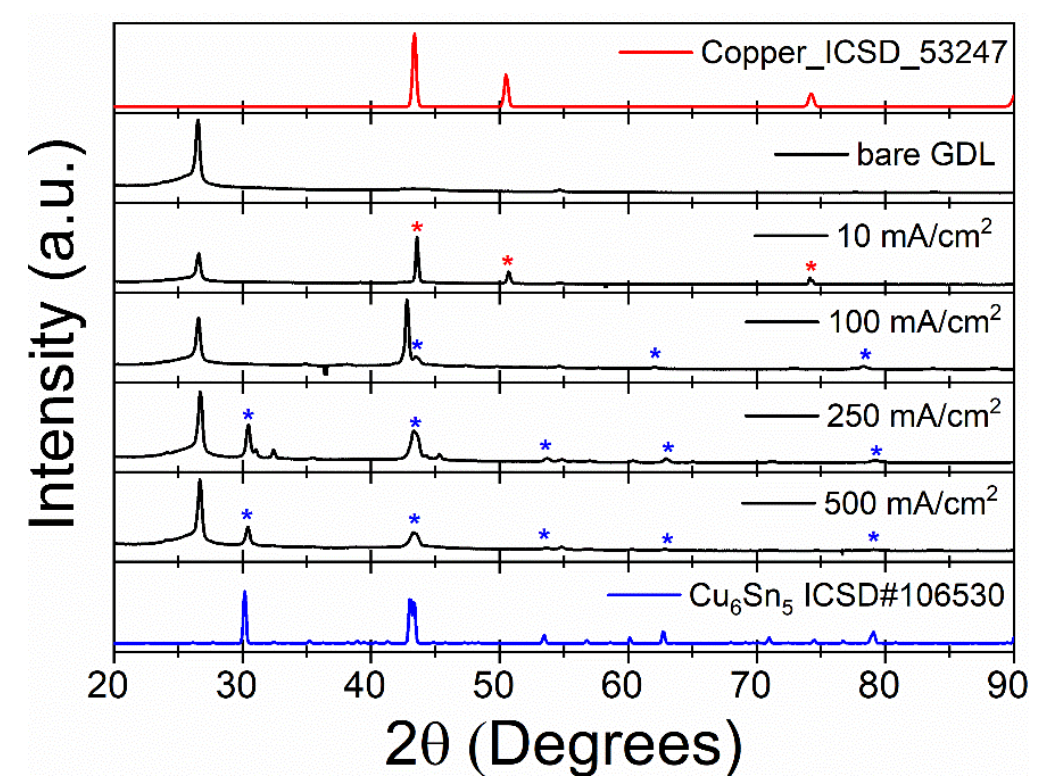

Figure 6. XRD patterns of samples electrodeposited at varying current densities. Appropriate reference standards with collection code are also included.

\section{Conclusions and Future Work}

Micro- and nano-structured deposits of $\mathrm{Cu}_{6} \mathrm{Sn}_{5}$ alloys were successfully electrodeposited on the surface of gas-diffusion layer substrates containing Teflon. A systematic investigation as a function of current density revealed a clear change in particle size as well as phase identity as confirmed by a both microscopy and X-ray diffraction studies. Future work will involve an expanded investigation of electrodeposition at lower and higher current densities, as well catalytic testing of these materials for the $\mathrm{eCO}_{2} \mathrm{RR}$.

\section{Acknowledgements}

Authors would like to thank WiSys AR-WiTAG grant, 2019- 2020, as well as the College of Science and Health for the Dean's Distinguished Fellowship grant. This material is based upon the work supported by NASA under award No. RIP20_3.0 issued through the Wisconsin Space Grant Consortium. Any opinions, findings and conclusions or recommendations expressed in this 
material are those of the authors and do not necessarily reflect the views of the National Aeronautics and Space Administration.

\section{References}

Chandrasekar, M. S., \& Pushpavanam, M. (2008). Pulse and pulse reverse plating - Conceptual, advantages and applications. Electrochimica Acta, 53(8), 3313-3322. doi: http://dx.doi.org/10.1016/j.electacta.2007.11.054

Hori, Y., Murata, A., \& Takahashi, R. (1989). Formation of hydrocarbons in the electrochemical reduction of carbon dioxide at a copper electrode in aqueous solution. Journal of the Chemical Society, Faraday Transactions 1: Physical Chemistry in Condensed Phases, 85(8), 2309-2326. doi: 10.1039/F19898502309

Hori, Y., Wakebe, H., Tsukamoto, T., \& Koga, O. (1994). Electrocatalytic process of CO selectivity in electrochemical reduction of $\mathrm{CO}_{2}$ at metal electrodes in aqueous media. Electrochimica Acta, 39(11-12), 1833-1839. doi: http://dx.doi.org/10.1016/0013-4686(94)85172-7

Katoh, A., Uchida, H., Shibata, M., \& Watanabe, M. (1994). Design of Electrocatalyst for $\mathrm{CO}_{2}$ Reduction: $\mathrm{V}$. Effect of the Microcrystalline Structures of Cu-Sn and $\mathrm{Cu}-\mathrm{Zn}$ Alloys on the Electrocatalysis of Reduction. Journal of The Electrochemical Society, 141(8), 2054-2058. doi: 10.1149/1.2055059

Low, C. T. J., \& Walsh, F. C. (2008). Electrodeposition of tin, copper and tin-copper alloys from a methanesulfonic acid electrolyte containing a perfluorinated cationic surfactant. Surface and Coatings Technology, 202(8), 1339-1349. doi: https://doi.org/10.1016/j.surfcoat.2007.06.032

Peterson, A. A., Abild-Pedersen, F., Studt, F., Rossmeisl, J., \& Norskov, J. K. (2010). How copper catalyzes the electroreduction of carbon dioxide into hydrocarbon fuels. Energy \& Environmental Science, 3(9), 1311-1315. doi: 10.1039/C0EE00071J

Sen, S., Brown, S. M., Leonard, M., \& Brushett, F. R. (2019). Electroreduction of carbon dioxide to formate at high current densities using tin and tin oxide gas diffusion electrodes. Journal of Applied Electrochemistry, 49(9), 917-928. doi: 10.1007/s10800-019-01332-z

Sen, S., Liu, D., \& Palmore, G. T. R. (2014). Electrochemical Reduction of $\mathrm{CO}_{2}$ at Copper Nanofoams. ACS Catalysis, 4(9), 3091-3095. doi: 10.1021/cs500522g

Sen, S., Skinn, B., Hall, T., Inman, M., Taylor, E. J., \& Brushett, F. R. (2016). Pulsed Electrodeposition of Tin Electrocatalysts onto Gas Diffusion Layers for Carbon Dioxide Reduction to Formate. MRS Advances, 2(8), 451-458. doi: 10.1557/adv.2016.652

Yin, Z., Palmore, G. T. R., \& Sun, S. (2019). Electrochemical Reduction of $\mathrm{CO}_{2}$ Catalyzed by Metal Nanocatalysts. Trends in Chemistry, 1(8), 739-750. doi: 10.1016/j.trechm.2019.05.004 